\title{
Effect of sequential radiofrequency ablation lesions at fast and slow atrioventricular nodal pathway positions in patients with paroxysmal atrial fibrillation
}

\author{
Clifford J Garratt, J Douglas Skehan, Gillian E Payne, Peter J Stafford
}

\begin{abstract}
Objective-To examine the hypothesis that the anatomic equivalents of the fast and slow pathways identified in patients with atrioventricular (AV) nodal tachycardia may be universal and represent the principal sites of atrial input into the normal compact $A V$ node.

Methods-15 patients undergoing complete AV junction ablation for paroxysmal atrial fibrillation were studied. Radiofrequency energy was delivered first in the anterior "fast pathway" position so as to prolong the atrium to bundle of His (AH) interval by over $50 \%$ of baseline (protocol 1) and then to the "slow pathway" position using the anatomical technique (protocol 2).
\end{abstract}

Results-Ablation protocol 1 resulted in prolongation of AH interval in all patients. Subsequent lesions at the level of the coronary sinus produced complete heart block in four patients, and in five caused a further increase in AH interval above that produced by protocol 1 . Four of these latter patients developed complete block after delivery of RF energy slightly anterior to the level of the coronary sinus os, as did three further patients in whom ablation at the level of the coronary sinus had no effect. In four patients complete heart block could not be achieved by protocol 2 .

Conclusions-A discrete anterior "fast" pathway and a posterior "slow" pathway or network of posterior pathways form the principal inputs to the compact AV node in most patients with atrial fibrillation. The absence of dual AV nodal physiology in the majority of these patients may be related to the functional properties of the individual components of this posterior network.

Academic Department of Cardiology,

Glenfield Hospital,

Groby Road, Leicester,

United Kingdom

C J Garratt

J D Skehan

G E Payne

P J Stafford

Correspondence to:

Dr C J Garratt, Academic Department of Cardiology, Glenfield Hospital, Groby Road, Leicester LE3 9QP,

(Heart 1996;75:502-508)

Keywords: atrioventricular node; catheter ablation; atrial fibrillation.

The application of catheter ablation techniques to patients with atrioventricular (AV) nodal reentrant tachycardia has been accompanied by an increased understanding of the anatomy and physiology of the human AV node. ${ }^{12}$ The fast AV nodal pathway of such patients can be selectively destroyed by lesions applied anteriorly, near the apex of Koch's triangle, resulting in a prolongation of $\mathrm{AV}$ node conduction time. ${ }^{34}$ The slow AV nodal pathway is ablated by lesions applied posteriorly, adjacent to the ostium of the coronary sinus. ${ }^{56}$ Anatomical studies of the AV node in humans with AV nodal reentrant tachycardia or dual AV nodal physiology have shown that the node and perinodal tissues of these patients are structurally no different from those of normal individuals, ${ }^{7}$ the implication being that the anatomical equivalents of the fast and slow pathways may be universal and represent the principal sites of atrial input into the normal compact AV node. The aim of our study was to examine this hypothesis by determining the effects of sequential radiofrequency (RF) catheter ablation lesions delivered first to the "fast pathway" and then to the "slow pathway" positions in patients without AV nodal reentrant tachycardia.

\section{Methods}

PATIENT SELECTION

Patients referred to our institution in a consecutive series for complete ablation of the AV junction were asked to participate in the study. The indication for this procedure in all patients was recurrent or established atrial fibrillation with symptoms uncontrolled by drug therapy. Ethical approval for the study was obtained from the local ethics committee and informed consent was obtained from all patients.

All patients underwent 12-lead electrocardiography, chest radiography, and transthoracic echocardiography on the day of admission to establish the rhythm at that time and the presence and extent of structural heart disease. Patients who were not in sinus rhythm on the day of admission to hospital underwent DC cardioversion, as is our usual clinical practice. Two patients were excluded from the trial because of established atrial fibrillation that did not respond to cardioversion. No other patients were excluded from the study.

BASELINE ELECTROPHYSIOLOGICAL STUDY All patients underwent a baseline electrophysiological study in the fasting state immediately before the ablation procedure. The electrophysiology study was performed with four electrode catheters (right ventricle, right atrium, His bundle, proximal coronary sinus) introduced through the femoral and subclavian veins. Multiple surface ECG leads and 
intracardiac electrograms from the different sites were simultaneously displayed and recorded on a multichannel oscilloscopic recorder and on paper at a speed of $100 \mathrm{~mm} / \mathrm{s}$. Atrioventricular nodal function was assessed using standard methods, with up to two right atrial extrastimuli being introduced during construction of antegrade curves at two basic drive cycles. Atrium to His bundle (AH) interval was measured from the earliest rapid deflection of the atrial electrogram on the His bundle catheter to the onset of the His bundle potential. Dual AV nodal pathways were considered to be present when there was more than a $50 \mathrm{~ms}$ increase in $\mathrm{AH}$ time following a $10 \mathrm{~ms}$ reduction in A1-A2 or A2-A3 interval. Ventriculo-atrial conduction was also assessed using standard techniques, with up to two right ventricular extrastimuli being introduced during construction of retrograde curves at two basic drive cycles. Patients proceeded to the ablation protocol irrespective of the presence or absence of dual AV nodal pathways.

\section{CATHETER ABLATION PROTOCOL}

All patients with a baseline $\mathrm{AH}$ interval of $<100 \mathrm{~ms}$ in sinus rhythm underwent ablation protocols 1 ("fast pathway" ablation) and 2 ("slow pathway" ablation) of the study. Patients with an initial AH interval of $>100 \mathrm{~ms}$ were considered to have no "fast pathway" antegrade conduction at baseline and underwent protocol 2 only.

\section{Protocol 1}

A 7F quadripolar steerable electrode catheter with a $4 \mathrm{~mm}$ distal electrode (Electrophysiology Technologies) was introduced percutaneously into the femoral vein, advanced to the right atrium, and positioned across the tricuspid valve so as to record the maximum $\mathrm{His}$ bundle potential. The catheter was then withdrawn several millimetres proximally until the atrial:ventricular electrode amplitude ratio recorded at the catheter tip was $>1$ and the amplitude of the His bundle electrogram was $<100 \mu \mathrm{V}$ (anatomical fast pathway position). Radiofrequency energy was then delivered (from a HAT 200 generator, Osypka) between the distal tip electrode and an indifferent patch electrode positioned on the chest according to the modified protocol of Langberg. ${ }^{8}$ Initially radiofrequency energy was delivered at a power output of $10 \mathrm{~W}$ for 10 to $15 \mathrm{~s}$. If no effects were seen, power output was increased by 2 to $4 \mathrm{~W}$ every 10 to $15 \mathrm{~s}$ up to a maximum of $30 \mathrm{~W}$. When junctional ectopy or an increase in the PR interval was seen, no further increments in power were made and the power output was continued at this same level for an additional 10 to $15 \mathrm{~s}$. At this time RF energy delivery was terminated and the $\mathrm{AH}$ interval in sinus rhythm was reassessed. If the $\mathrm{AH}$ interval had not increased by at least $50 \%$ over the baseline value, then the catheter was repositioned and radiofrequency energy delivery repeated according to the protocol described above. In contrast, if the $\mathrm{AH}$ interval was increased by $50 \%$ or more over the baseline value and remained so after a $5 \mathrm{~min}$ waiting period, no more energy was delivered in the "fast pathway" position (end point for protocol 1). After completion of protocol 1, $\mathrm{AV}$ nodal function was reassessed in both antegrade and retrograde directions, as described for the initial baseline electrophysiology study. Particular attention was paid to the timing of retrograde activation of the atrium at the proximal coronary sinus relative to that at the His bundle catheter during construction of retrograde curves.

\section{Protocol 2}

The ablation catheter was slowly withdrawn along the tricuspid septal annulus down to the most posterior/inferior aspect of the interatrial septum adjacent to the coronary sinus ostium (region $\mathrm{P}$ on fig 1). The site was considered optimal if the bipolar recording obtained from the distal electrodes showed an atrial:ventricular electrogram ratio of $0 \cdot 1$ to $0 \cdot 5$. The presence of slow pathway potentials was sought but was not considered a prerequisite for energy delivery at such a site. Radiofrequency energy was delivered at this position at a power output of $30 \mathrm{~W}$ until complete heart block was achieved or until $30 \mathrm{~s}$ had elapsed. Radiofrequency energy delivery was terminated prematurely if junctional ectopy was not observed, suggesting the presence of poor tissue contact or if a marked rise in impedance occurred. If complete heart block did not result after $30 \mathrm{~s}$ of energy delivery then, while an optimal $\mathrm{A}: \mathrm{V}$ ratio was maintained, the catheter tip was moved a few millimetres anteriorly and RF energy redelivered according to the protocol of slow pathway ablation described by Jazayeri et al. ${ }^{6}$ The end points of protocol 2 were complete heart block or the recording of a His bundle electrogram on the ablation catheter.

Those patients that were not in complete heart block at the end of protocol 2 underwent standard His bundle ablation. After evaluation of the underlying escape rhythm, all patients

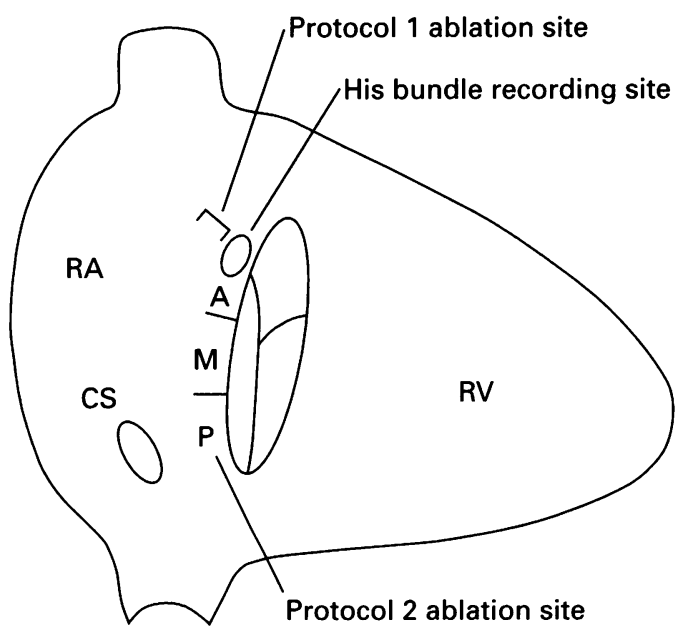

Figure 1 Schematic representation of ablation sites for protocols 1 and 2 (right anterior oblique view of tricuspid annulus). The atrioventricular junction of the septal cusp is arbitrarily divided into three regions: posterior $(P)$ medial $(M)$, and anterior $(A)$. CS, coronary sinus; $R A$, right atrium; $R V$, right ventricle. 
Clinical and electrophysiological characteristics, and effects of ablation protocols 1 and 2

\begin{tabular}{|c|c|c|c|c|c|c|c|c|c|c|c|c|c|}
\hline \multirow[b]{3}{*}{ Patient } & \multirow{3}{*}{$\begin{array}{l}\text { Agel } \\
\text { sex }\end{array}$} & \multirow{3}{*}{$\begin{array}{l}\text { AF } \\
\text { duration } \\
\text { (years) }\end{array}$} & \multirow{3}{*}{$\begin{array}{l}\text { Drugs } \\
\text { tried (n) }\end{array}$} & \multicolumn{4}{|l|}{ Baseline } & \multicolumn{3}{|c|}{ Protocol 1} & \multirow{2}{*}{\multicolumn{3}{|c|}{ Effect of ablation protocol 2}} \\
\hline & & & & \multirow{2}{*}{$\begin{array}{l}\text { AH } \\
\text { interval } \\
\text { (ms) }\end{array}$} & \multirow{2}{*}{$\begin{array}{l}A V \\
\text { nodal } \\
\text { Duality }\end{array}$} & \multirow{2}{*}{$\begin{array}{l}A V N \\
A E R P \\
(m s)\end{array}$} & \multirow{2}{*}{$\begin{array}{l}\text { Retrograde } \\
\text { conduction }\end{array}$} & \multirow{2}{*}{$\begin{array}{l}\text { AH } \\
\text { interval } \\
\text { (ms) }\end{array}$} & \multirow{2}{*}{$\begin{array}{l}A V N \\
A E R P \\
\text { (ms) }\end{array}$} & \multirow{2}{*}{$\begin{array}{l}\text { Retrograde } \\
\text { conduction }\end{array}$} & & & \\
\hline & & & & & & & & & & & Region & Region $M$ & Region $A$ \\
\hline 1 & $66 \mathrm{M}$ & 20 & 6 & 110 & No & 280 & No & - & - & - & $\mathrm{AH} \geqslant 150 \mathrm{~ms}$ & $\mathrm{CHB}$ & - \\
\hline 2 & $65 \mathrm{M}$ & 5 & 5 & 100 & No & 430 & No & - & - & - & $\mathrm{AH} \geqslant 170 \mathrm{~ms}$ & No effect & No effect \\
\hline 3 & $66 \mathrm{~F}$ & 5 & 5 & 80 & No & 250 & Yes & 135 & NP & No & No effect & No effect & No effect \\
\hline 4 & $70 \mathrm{M}$ & 9 & 5 & 130 & No & 250 & No & - & - & - & $\mathrm{CHB}$ & - & - \\
\hline 5 & $60 \mathrm{M}$ & 15 & 6 & 40 & No & 220 & Yes & 110 & NP & Yes & CHB & - & - \\
\hline 6 & $63 \mathrm{M}$ & 10 & 6 & 60 & No & 200 & Yes & 100 & 200 & Yes & No effect & CHB & - \\
\hline 7 & $51 \mathrm{M}$ & 26 & 6 & 75 & No & 260 & No & 155 & 260 & No & $\mathrm{CHB}$ & & - \\
\hline 8 & $50 \mathrm{~F}$ & 10 & 6 & 120 & No & 280 & No & - & - & - & $\mathrm{AH} \geqslant 200 \mathrm{~ms}$ & CHB & - \\
\hline 9 & $66 \mathrm{~F}$ & 20 & 6 & 75 & No & 230 & No & 160 & 220 & No & No effect & No effect & No effect \\
\hline 10 & $67 \mathrm{M}$ & 6 & 6 & 60 & No & 250 & Yes & 120 & 250 & No & $\mathrm{AH} \geqslant 150 \mathrm{~ms}$ & $\mathrm{CHB}$ & - \\
\hline 11 & $74 \mathrm{~F}$ & 3 & 5 & 85 & No & 250 & No & 140 & 240 & No & $\mathrm{AH} \geqslant 185 \mathrm{~ms}$ & $\mathrm{CHB}$ & - \\
\hline 12 & $73 \mathrm{~F}$ & 3 & 3 & 75 & No & 270 & Yes & 120 & 280 & No & No effect & No effect & No effect \\
\hline 13 & $53 \mathrm{M}$ & 5 & 4 & 45 & Yes & 340 & Yes & 100 & 360 & Yes & $\mathrm{CHB}$ & - & - \\
\hline 14 & $47 \mathrm{M}$ & 3 & 7 & 85 & No & 240 & Yes & 130 & 230 & No & No effect & CHB & - \\
\hline 15 & $74 \mathrm{~F}$ & 6 & 4 & 75 & Yes & 220 & Yes & 120 & 260 & No & No effect & $\mathrm{CHB}$ & - \\
\hline
\end{tabular}

$\overline{\mathrm{AF}}$, atrial fibrillation; AVN AERP, atrioventricular nodal antegrade effective refractory period; CHB, complete heart block; -, not applicable; NP, not performed; M, male; F, female.

underwent implantation of a permanent pacemaker. The total number of RF deliveries in each protocol and the anatomical position of the ablation catheter at sites of RF energy delivery were recorded for all patients.

\section{ANALYSIS OF RESULTS}

All values are expressed as mean (SD). A difference between paired samples was considered statistically significant when $P$ was less than 0.05 according to the Student's $t$ test.

\section{Results}

PATIENT CHARACTERISTICS

The clinical and electrophysiological characteristics of the 15 patients that took part in the study are detailed in the table. Mean age was 63 years (range 47-74); six were female and nine were male. Structural heart disease was evident on echocardiography in 13 patients: left ventricular dilatation in seven (three had previously documented coronary artery disease), and left atrial dilatation in eight. One patient had previous mitral valve replacement for rheumatic heart disease and another had mild (unoperated) mitral stenosis. One patient had a previous ASD repair with evidence of

Figure 2 The effect of ablation protocol 1 on $A H$ interval during sinus rhythm.

\section{Baseline}

(1)

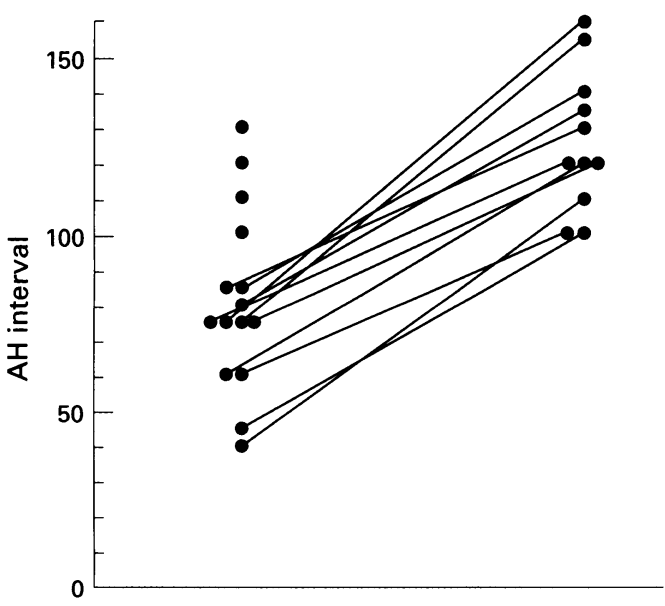

right atrial and ventricular dilatation. The mean time from first documented occurrence of AF was 9.7 years (range 3 to 26) and a mean of 5.3 drugs (range 3 to 7) had been tried in an attempt to improve symptoms or maintain sinus rhythm. The mean PR interval at baseline in sinus rhythm was $0.18 \mathrm{~s}$ (range $0 \cdot 10$ to $0.24 \mathrm{~s}$ ). One patient (patient No 1) had undergone a previous unsuccessful attempt at standard His bundle ablation that had resulted in an increase in PR interval from 0.16 to $0.22 \mathrm{~s}$. Three patients had been thought to be in established atrial fibrillation until cardioverted to sinus rhythm the day before the procedure. At baseline electrophysiological study, only two patients fulfilled the criteria for antegrade AV nodal duality and none had inducible junctional reentrant tachycardias of any type. At baseline mean $\mathrm{AH}$ interval was $81 \mathrm{~ms}$ (range 40 to $130 \mathrm{~ms}$ ) and mean AV nodal antegrade effective refractory period was $264 \mathrm{~ms}$ (range 200 to $430 \mathrm{~ms}$ ). Retrograde conduction was present in only eight patients at a ventricular paced cycle length of 550 or 600 ms. During ventricular pacing, the earliest atrial activation occurred at the His bundle electrode position in seven of these patients and at the proximal coronary sinus in one patient (No 13): ventriculo-atrial conduction showed decremental properties in all eight patients.

\section{EFFECTS OF PROTOCOL 1 ("FAST PATHWAY"} ABLATION)

All 11 patients with a baseline $\mathrm{AH}$ interval $<100 \mathrm{~ms}$ in sinus rhythm underwent ablation protocol 1, resulting in an increase in mean $\mathrm{AH}$ interval of $58 \mathrm{~ms}$ (mean increase of $84 \%$ over baseline value) in these patients (see fig 2). Although a mean of 3.5 ablation lesions per patient (range 1 to 11 ) was delivered, the prolongation of $\mathrm{AH}$ interval occurred as an "all or none" phenomenon after a single RF lesion in all cases, that is, it was not the result of a succession of smaller AH changes. Among the nine patients in whom it was measured both before and after protocol 1 , antegrade effective refractory period of the AV node was not significantly affected $(251 \mathrm{~ms} v 255 \mathrm{~ms}$, 
NS). All of the eight patients with intact ventriculo-atrial conduction at baseline underwent ablation protocol 1: retrograde conduction block developed in five of these patients as a result of this protocol. In two patients there was an increase in VA interval associated with a shift in earliest atrial activation from that recorded at the His bundle catheter to that at the proximal coronary sinus, and in one patient (No 13) VA conduction remained unaffected.

EFFECTS OF PROTOCOL 2 ("SLOW PATHWAY" ABLATION)

All 15 patients underwent this part of the study (see table). Lesions were delivered initially at the level of the coronary sinus os (region $P$ in fig 1 ) in all cases. Ablation at this level caused complete heart block in four of the 15 patients, further $\mathrm{AH}$ prolongation without complete block in five, and had no obvious effect in the remaining six patients (mean of $2 \cdot 1$ lesions in region $P$, range 1 to 3 ). Radiofrequency ablation lesions were then delivered to the mid position (region $M$ in fig 1) in all 11 patients who had not developed complete heart block following posterior ablation. Complete heart block occurred after mid-position ablation in seven of these 11 patients, including four of the five patients who had $\mathrm{AH}$ prolongation after posterior lesions (mean of 2.5 lesions in region $M$, range 1 to 6). In the remaining four patients with intact AV conduction, ablation lesions were applied in the anterior position (region $\mathrm{A}$ in fig 1) but in no case did complete block occur (mean of $2 \cdot 2$ lesions, range 2 to 3 ). In these patients standard His bundle ablation was performed. Success or failure of protocols 1 and 2 in causing complete heart block was not associated with any identifiable baseline characteristic (AH interval, AV nodal effective refractory period, presence of retrograde conduction). A mean of five RF deliveries per patient (all regions) was given (range 1 to 12 ) in protocol 2.

\section{PROPERTIES OF ESCAPE RHYTHM AFTER}

ABLATION

Of the four patients who underwent conventional His bundle ablation, one had no detectable underlying ventricular rhythm at the end of the procedure. Of the 11 patients who developed complete block after proximal AV node ablation (sequential ablation at fast and then slow pathway positions), all had a detectable underlying narrow complex ventricular escape rhythm (mean cycle length 1445 $\mathrm{ms}$, range 1060 to 1800 ). The $\mathrm{HV}$ interval was unchanged from baseline value in the seven patients in whom it was recorded. Ten of the 11 patients who developed complete block after proximal AV nodal ablation were reevaluated at a mean of 31 weeks (range 8 to 60) after ablation. None had return of AV conduction and all had a narrow complex escape rhythm. Mean cycle length of the escape rhythm was not significantly altered from immediately after ablation, at $1417 \mathrm{~ms}$, range 1093 to 1832 .

\section{Discussion}

Our study has confirmed that delivery of RF energy in the "fast pathway" position results in $\mathrm{AH}$ prolongation in patients without dual AV nodal physiology, providing further evidence that under normal circumstances (in the absence of dual AV nodal physiology) earliest activation of the AV node during sinus rhythm occurs through an anterior atrio-nodal input. The fact that $\mathrm{AH}$ prolongation was a sudden all or none phenomenon rather than progressive over several successive lesions supports the concept of ablation of a specific anterior atrionodal input in these patients. The lack of effect of anterior ablation on AV nodal refractoriness, as measured by the extrastimulus technique, is in keeping with the findings after fast pathway ablation in patients with $A V$ nodal tachycardia. ${ }^{34}$ More importantly, our study has shown that sequential ablation of tissue in both the "fast" and "slow" pathway positions in patients with paroxysmal atrial fibrillation frequently results in complete $\mathrm{AV}$ block, suggesting that anterior and posterior atrio-nodal fibres may provide the principal atrial inputs to the compact AV node, even in patients without AV nodal reentrant tachycardia or demonstrable dual AV nodal physiology. If this were not the case it is difficult to see how complete antegrade block could have resulted from the lesions directed at these two sites alone. This finding is consistent with previous isolated reports of patients with $\mathrm{AV}$ nodal reentrant tachycardia who have developed complete heart block either from slow pathway ablation in the setting of "tenuous" antegrade fast pathway conduction ${ }^{9}$ or after attempts at both fast and slow pathway ablation at the same sitting. ${ }^{10}$

Previous studies of patients with atrial fibrillation have examined the effects of radiofrequency catheter ablation in either the anterior or posterior position alone. Duckeck and coworkers ${ }^{11}$ described a protocol in which they delivered radiofrequency lesions at a site similar to that described for fast pathway ablation, in an attempt to increase the AV nodal Wenkebach cycle length and improve symptomatic status without the requirement for permanent pacemaker implantation. The proposed endpoint of their protocol was a combination of $\mathrm{AH}$ prolongation and a Wenkebach cycle length of $>400 \mathrm{~ms}$, but this could be achieved in only 10 of 50 patients. Most patients developed $\mathrm{AH}$ prolongation alone (and recurrent symptoms) or complete heart block. In contrast, several recent reports have documented the ability of radiofrequency catheter ablation lesions directed at the posterior or midseptal regions to decrease ventricular rate without causing heart block in patients with atrial fibrillation. ${ }^{12-15}$ The protocol used by Williamson et al $^{14}$ is similar to the anatomical approach for slow pathway ablation described by Jazayeri et $a l^{6}$ and used in our study. The procedure was apparently successful in that it decreased ventricular rate to 120 beats/min during isoprenaline in 14 of the 19 patients studied (74\%). Feld and coworkers ${ }^{13}$ used a slightly different technique in which 
Figure 3 Possible explanations for the effects of sequential ablation in pathway" positions. ( $A$ ) In sinus rhythm activation of the His bundle occurs through the anterior atrionodal input. Ablation protocol 1 results in conduction block through this anterior input, and slow antegrade conduction over the posterior atrionodal input is revealed. Ablation at the level of the coronary sinus (region $P$ ) or more anteriorly (region $M$ ) causes conduction block in the posterior input and complete heart block results. (B) Ablation protocol 1 results in conduction block through anterior atrio-nodal input as in $(A)$; antegrade conduction occurs preferentially over the posterior input. Ablation of the posterior input at the level of the coronary sinus (region P) reveals conduction over a second, more anterior and more slowly conducting, entrance to the posterior input. This second entrance is blocked by ablation lesions anterior to the level of the coronary sinus (region $M$ ).
A

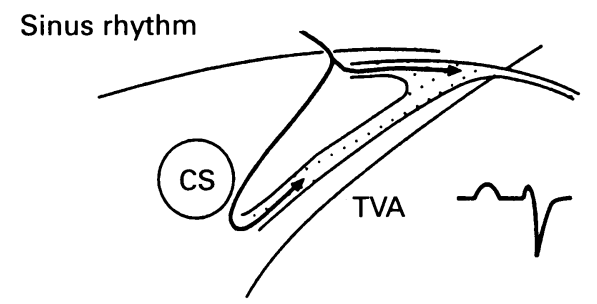

Protocol 1

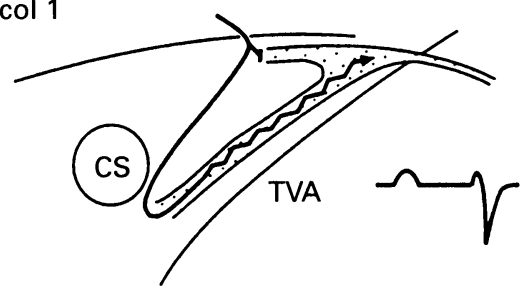

Protocol 2

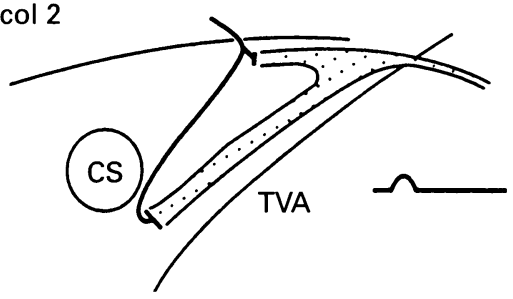

ablation lesions were applied initially at a midseptal position, with additional lesions applied progressively posteriorly until the ventricular response during atrial fibrillation fell below 100 beats/min. These studies suggest that AV nodal refractoriness (and ventricular rate in $\mathrm{AF}$ ) is determined primarily by a posterior atrio-nodal input similar to the slow pathway seen in patients with AV nodal reentrant tachycardia. Other studies suggest that there are differences between the two patient groups, however. Della Bella et al ${ }^{15}$ have reported that prolongation of AV nodal refractoriness induced by this procedure in patients with atrial fibrillation is a stepwise phenomenon requiring energy delivery at several sites, rather than the all or none response of slow pathway ablation in patients with AV nodal tachycardia or "fast pathway" ablation seen in our study. With the relatively low success rate of the procedure in patients with AF (compared with AV nodal reentrant tachycardia), this may indicate that the posterior input is more diffuse or is more of a network than a discrete pathway in these patients.

Of particular interest in this regard is the response of four patients in our study in whom ablation at the level of the coronary sinus os produced $\mathrm{AH}$ prolongation additional to that seen after initial fast pathway ablation, but in whom complete block was achieved by ablation slightly anterior to the original slow pathway ablation site. The presence of a second or multiple posterior inputs or multiple entrances to a single posterior input (fig 3) are possible explanations of this phenomenon. Residual
B

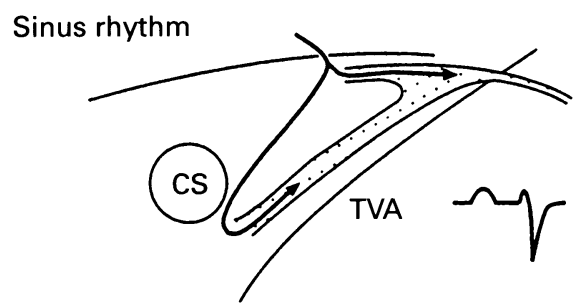

Protocol 1

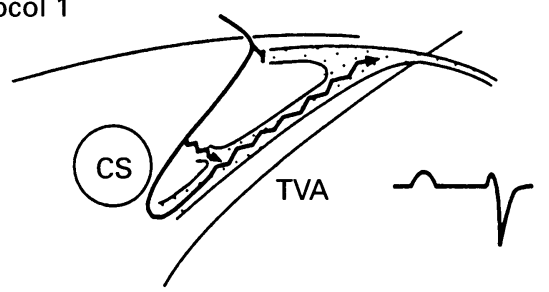

Protocol 2

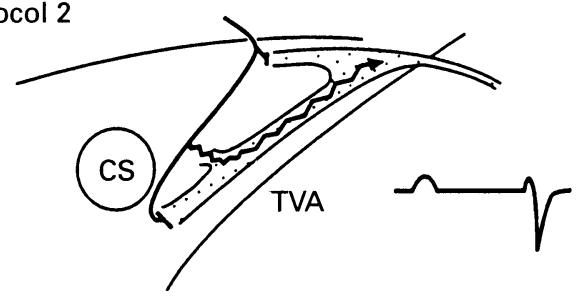

slow pathway conduction is not uncommon after successful slow pathway ablation for AV nodal reentrant tachycardia ${ }^{6}$ and would also be in keeping with the concept of multiple posterior inputs. These clinical observations are perhaps not surprising given that multiple atrionodal bundles (transitional cell zones) have been demonstrated in anatomical studies. ${ }^{16}$ It is possible that the relative refractoriness of the various entrances to the main posterior input (or of separate posterior inputs) may be the main determinant of the presence or absence of dual AV nodal physiol${ }^{0 g y}{ }^{17}$ in these patients. Further elucidation of the precise physiology of the atrionodal inputs to the compact node may require detailed intracardiac mapping using intraoperative multielectrode techniques: such studies would avoid the problems of interpretation inherent in the use of catheters with electrodes at varying orientations and distances from the endocardial surface. The question of whether ablation lesions at slow pathway sites (posterior or mid locations) might produce their effects by causing partial damage to the compact AV node has been addressed by several workers and anatomical studies would suggest that this is unlikely, ${ }^{2}$ although it cannot be definitively excluded. The heterogeneity of AV nodal anatomy is such that varying degrees of heart block can result from lesions at these sites in patients with AV nodal reentrant tachycardia, but in practice it is uncommon (less than $2 \%$ ), even using a purely anatomical approach. Histopathological examination of a single patient following slow pathway ablation pro- 
vides supportive evidence that such lesions do not involve the compact $\mathrm{AV}$ node. ${ }^{18}$

The study protocol was specifically designed so that fast pathway ablation was performed before slow pathway ablation, and in no instance was slow pathway ablation performed first. The incidence of complete heart block associated with the fast pathway approach alone (at least in patients with AV nodal tachycardia) is sufficiently high in some series for the occurrence of complete block with a sequential "slow then fast" pathway ablation protocol to be very difficult to interpret: it could always be said to be the result of overzealous fast pathway ablation. In addition, the end point for slow pathway ablation alone in patients with characteristics similar to our study group is very poorly defined: most studies of this approach have been performed while the patients are in atrial fibrillation. ${ }^{1314}$ In our study we followed a stepwise "anatomical" approach to slow pathway ablation, rather than being guided by the presence of slow pathway potentials. Recent intraoperative multielectrode studies of the triangle of $\mathrm{Koch}^{19}$ have suggested that high or low frequency potentials are relatively non-specific guides to ablation and may be found at regions remote from, as well as at the site of, the earliest retrograde atrial exit of the slow pathway. It may be that a combination of slow potential localisation and retrograde atrial mapping is the optimal approach, ${ }^{5}$ but retrograde conduction by the slow pathway was present in only three of the 15 patients in our study.

Sequential fast and slow pathway ablation was unsuccessful in causing complete antegrade block in four of the 15 patients $(27 \%)$. This failure rate is similar to that described for posterior ablation in previous studies of patients with atrial fibrillation. ${ }^{1314}$ It may be that the reasons for failure are the same as proposed for these studies of posterior ablation alone, that is, the presence of a network of posterior atrionodal inputs rather than a single discrete pathway. Alternatively, inputs from the left atrium may be important in some patients. The anterior and posterior atrionodal fibres are formed from the transitional cell group. The anterior group comprises in turn both superficial and deep fibres. The deep fibres approach the compact node from the left side of the atrial septum ${ }^{16}$ and, unlike the superficial fibres that originate in the right atrial aspect of the muscular rim of the oval fossa, are unlikely to be destroyed by anterior ablation lesions. Finally it is possible that failure to perform protocol 1 contributed to the failure of protocol 2 to cause complete heart block in one patient (No 2), that is, antegrade fast pathway conduction may have been present despite a relatively long $\mathrm{AH}$ time and the absence of retrograde conduction at baseline.

STUDY LIMITATIONS

As a result of the nature of the selection process, the subjects entered into this study were far from "normal" in terms of cardiac structure and AV nodal function. In addition to the almost universal presence of structural heart disease, $\mathrm{AH}$ interval was prolonged, and retrograde (ventriculo-atrial) conduction was absent at baseline in a high proportion of cases. Consequently one cannot automatically assume that the findings of this study would be reproduced if the protocol were to be performed using subjects with completely normal hearts. Nevertheless, the fact that success or failure of the protocol to achieve complete heart block was not associated with any identifiable abnormal baseline characteristic would suggest that this is likely to be the case.

As previously stated, this study is unusual for this patient group in that the majority of subjects were in sinus rhythm during the assessment, allowing confirmation of the presence or absence of dual AV nodal physiology as well as a comparison of antegrade AV nodal refractoriness (as assessed by the extrastimulus technique) before and after fast pathway ablation. Incremental atrial pacing (in order to determine the shortest atrial pacing interval associated with 1:1 AV conduction) was not performed, either before or after delivery of ablation lesions, so as to minimise the risk of induction of atrial fibrillation. Results of the extrastimulus technique, although not strictly equivalent, are closely correlated with those of incremental pacing techniques both in patients with atrioventricular nodal reentrant tachycardia after fast pathway ablation ${ }^{346}$ and in unselected patients undergoing electrophysiological assessment. ${ }^{20}$

\section{CLINICAL RELEVANCE}

These findings have clinical relevance in so far as they show that a "proximal" approach to AV nodal ablation is an effective means of creating complete heart block in patients with atrial fibrillation. The merits or otherwise of this technique relative to the more standard (distal) approach to AV node ablation were not addressed by this study.

Dr Stafford is supported by the British Heart Foundation Grant No PG 93/105)

1 Akhtar M, Jazayeri MR, Sra J, Blanck Z, Deshpande S, Dhala A. Atrioventricular nodal reentry: clinical, electrophysiological and therapeutic considerations. Circulation 1993;88:282-95.

2 McGuire MA, Janse MJ, Ross DI "AV nodal" reentry: AV nodal, AV junctional or atrionodal reentry? $f$ Cardiovasc Electrophysiol 1993;4:573-86.

3 Haissaguerre M, Warin JF, Lemetayer P, Saoudi N, Guillem JP, Blanchot P. Closed-chest ablation of retrograde conduction in patients with atrioventricular nodal grade conduction in patients with atrioventricular nod
reentrant tachycardia. N Eng fMed 1989;320:426-33.

4 Lee MA, Morady F, Kadish A, Schamp DJ, Chin MC, Scheinman MM, et al. Catheter modification of the atrioventricular junction with radiofrequency energy for control of atrioventricular nodal reentry tachycardia. Circulation 1991;83:827-35.

5 Jackman WM, Beckman KJ, McClelland JH, Wang X, Friday KJ, Roman CA, et al. Treatment of supraventricular tachycardia due to atrioventricular nodal reentry by radiofrequency catheter ablation of slow pathway conduction. $N$ Eng $\mathcal{F}$ Med 1992;327:313-8.

6 Jazayeri MR, Hempe SL, Sra JS, Dhala AA, Blank A Deshpande SS, et al. Selective transcatheter ablation of the fast and slow pathways using radiofrequency energy in patients with atrioventricular nodal reentrant tachycardia. Circulation 1992;85:1318-28.

$7 \mathrm{Ho}$ SY, McComb JM, Scott CD, Anderson RH. Morphology of the cardiac conduction system in patients with electrophysiologically proven dual atrioventricular nodal pathways. If Cardiovasc Electrophysiol 1993;4: 504-12.

8 Langberg JJ. Radiofrequency catheter ablation of AV nodal reentry: the anterior approach. $P A C E$ 1993;16:615-22.

9 Langberg JJ, Leon A, Borganelli M, Kalbfleisch SJ, ElAtassi $\mathrm{R}$, Calkins $\mathrm{H}$, et al. A randomised, prospective comparison of anterior and posterior approaches to 
radiofrequency catheter ablation of atrioventricular nodal reentry tachycardia. Circulation 1993;87:1551-6.

10 Hindricks G. The Multicentre European Radiofrequency Survey [MERFS]: complications of radiofrequency catheter ablation of arrhythmias. Eur Heart $\mathcal{f}$ 1993;14: catheter $1644-53$.

11 Duckeck W, Engelstein ED, Kuck K-H. Radiofrequency current therapy in atrial tachyarrhythmias: modulation versus ablation of atriove

2 Fleck RP, Chen P-S, Boyce K, Ross R, Dittrich HC, Feld GK. Radiofrequency modification of atrioventricular conduction by selective ablation of the low posterior septal right atrium in a patient with atrial fibrillation and a rapid ventricular response. $P A C E$ 1993;16:377-81.

13 Feld GK, Fleck RP, Fujimara O, Prothro DL, Bahnson TD, Ibarra M. Control of rapid ventricular response by radiofrequency catheter modification of the atrioventricular node in patients with medically refractory atrial fibrillation. Circulation 1994;90:2299-307.

14 Williamson BD, Man KC, Daoud E, Niebauer M, Strickberger SA, Morady F. Radiofrequency catheter ventricular rate during atrial fibrillation. $N$ Eng $f \mathrm{Med}$ 1994;331:910-17.
15 Della Bella P, Carbucicchio C, Tondo C, Riva S. Modulation of atrioventricular conduction by ablation of the "slow" atrioventricular node pathway in patients with drug-refractory atrial fibrillation or flutter. $\mathcal{F} \mathrm{Am}$ Coll Cardiol 1995;25:39-46.

16 Anderson RH, Becker AE, Brechenmacher C, Davies M, Rossi L. The human atrioventricular junctional area. A Rossi L. The human atrioventricular junctional area. A
morphological study of the AV node and bundle. Eur $\mathcal{f}$

17 Moe G, Preston J. Physiologic evidence for a dual AV transmission system. Circ Res 1956;4:357-75.

8 Gamache $M$, Bharati $S$, Lev $M$, Lindsay BD. Histopathological study following catheter guided radiofrequency current ablation of the slow pathway in a patient with atrioventricular nodal reentrant tachycardia. $P A C E$ 1994;17:247-51.

19 McGuire M, Bourke J, Robotin MC, Johnson DC, Meldrum-Hanna W, Nunn GR, et al. High resolution mapping of Koch's triangle using sixty electrodes in humans with atrioventricular junctional (AV nodal) reentrant tachycardia. Circulation 1993;88:2315-28.

20 Fisher JD, Zhang X, Waspe LE, Kim SG, Mercando AD. Tests of refractoriness and conduction during clinical electrophysiologic studies: yields and roles. $\mathcal{f}$ Electrophysiol 1988;2:175-89. 